[3] Sites, J. R., Lui, X.: Progress in Photovoltaics 3 (1995), S. 307 bis 314 .

[4] Tyan, Y.-S., Perez-Albuerne, E. A.: Conf. Record of the 16th Photovoltaic Specialists Conference 1982, S. 794 bis 800 .

[5] Bonnet, D., Rabenhorst, H.: Conf. Record of the 9th Photovoltaic Specialists Conf. 1972, S. 129 bis 131.

[6] Bonnet, D.: Proc. 14th European Photovoltaic Solar Energy Conf. 1997, S. 2688 bis 2693.

[7] Chu, T. L., Chu, Sh., Britt, J., Chen, G., Ferekides, C., Ullal, H. S.: Proc. 11th European Photovoltaic Solar Energy Conf. 1992, S. 988 bis 990.
[8] Ferekides, C., Marinski, D., Morel, D. L.: Conf. Record of the 26th Photovoltaic Specialists Conf. 1997, S. 339 bis 342 .

[9] Sasala, R. A., Powell, R. C., Dorer, D. L., Reiter, N.: NREL/SNL Photovoltaics Program Review Meeting, Nov. 18-22, Lakewood CO, USA, APS Conference Proceedings 394 (1996), S. 171 bis 188.

[10] Bonnet, D.: Int. J. Solar Energy 12 (1992), S. 1 bis 14.

[11] Marshall, J.: San Francisco Chronicle, 20. 5. 1997.

[12] -Meyers, P. V., Birkmire, W.: Progress in Photovoltaics. Research and Applications 3 (1995), 393 bis 402.

\title{
Zukunftschancen der Photovoltaik
}

\section{F. Vahrenholt ${ }^{1}$}

Eine Darstellung der Gründe, weshalb Shell die Photovoltaik zu einem ihrer Kerngeschäfte erklärte:

- Die Endlichkeit von Öl- und Gasressourcen wird im Verlauf des nächsten Jahrhunderts spürbar.

- Bevor Knappheiten zu erheblichen Verteuerungen führen, werden die durch $\mathrm{CO}_{2}$ und Methan ausgelösten Klimaveränderungen zu einer Forderung nach Drosselung der Verbrennung fossiler Brennstoffe führen.

- Regenerative Energien haben weltweit eine große Marktchance.

Schlüsselwörter: nachhaltiges Wachstum; Dematerialisierung; erneuerbare Energien; Photovoltaik

Future chances of photovoltaics. A presentation of reasons for Shell do declare photovoltaics to once of their main trades:

- The limitation of oil and gas resources will be notable in the course of the next century.

- Before the shortnesses provoke massive increases in costs, the changes in climate caused by $\mathrm{CO}_{2}$ and methane will lead to a demand of tapering off the burning of fossile fuels.

- Regenerative energies have globally a great market chance.

Keywords: lasting growth; dematerialization; renewable energies; photovoltaics

Die Jahre 1997 und 1998 werden vielleicht einmal als die Jahre des Beginns einer Erfolgsgeschichte einer neuen Energietechnologie, der Photovoltaik, angesehen werden. Seit Jahrzehnten ist diese Technologie bekannt, aber über ein Nischen- und Mauerblümchendasein kam sie nicht hinaus.

Drei Ereignisse markieren die Trendwenden:

\footnotetext{
${ }^{1}$ Dr. Fritz Vahrenholt, Vorstandsmitglied der Deutschen Shell AG, Überseering 35, D-22297 Hamburg.
}

(1) Kyoto als Startpunkt einer globalen Zielsetzung der Entwicklung regenerativer Energien,

(2) das Weißbuch der EU mit dem ehrgeizigen Ziel bis $20103000 \mathrm{MW}$ installierte Leistung allein in Europa,

(3) und nicht zuletzt der Weltkongreß der Photovoltaik, der gezeigt hat, Photovoltaik wird ein ,core business" großer mutlinationaler Unternehmen von Siemens über Kyocerca, BP und Shell. 
Warum erklärt ein Unternehmen wie die Shell Photovoltaik zum Kerngeschäft?

(1) Die Endlichkeit von Öl- und Gasressourcen wird im Verlauf des nächsten Jahrhunderts spürbar.

(2) Bevor Knappheiten zu erheblichen Verteuerungen führen, werden die durch $\mathrm{CO}_{2}$ und Methan ausgelösten Klimaveränderungen $\mathrm{zu}$ einer Forderung nach Drosselung der Verbrennung fossiler Brennstoffe führen.

(3) Regenerative Energien haben weltweit eine große Marktchance.

Die Shell-Gruppe ist für ihre weitreichenden Szenarien bekannt. Im Bereich des Energieverbrauchs wurden zwei Szenarien - „Nachhaltiges Wachstum" und „Dematerialisierung“ - bis zum Jahr 2060 erarbeitet.

Beide Szenarien gehen von der gleichen Bevölkerungsentwicklung (zehn Milliarden Menschen im Jahre 2060 gegenüber fünf Milliarden in 1990) und von gleichem Wirtschaftswachstum ( $3 \%$ pro Jahr) aus. Der Weltenergieverbrauch verdreifacht sich im Szenario „Nachhaltiges Wachstum“, im anderen Fall verdoppelt er sich. In beiden Szenarien werden sich die regenerativen Energien im Rahmen eines Reifeprozesses weiterentwickeln, indem sie zunächst Marktnischen besetzen und sich dann mit unterschiedlichem Erfolg im Markt durchsetzen. Im Szenario „Nachhaltiges Wachstum" geht Shell davon aus, daß Produktivitätsfortschritte bei der Energiebereitstellung zu einem erheblichen Angebot an regenerativen Energieträgern führen wird. Konkret könnten erneuerbare Energiequellen bis zur Mitte des nächsten Jahrhunderts rund $50 \%$ des Weltenergiebedarfs decken (Abb. 1). Im Szenario ,Dematerialisierung“" sind es immerhin $30 \%$.

Ein Vergleich des Energieverbrauchs der industrialisierten Länder mit den Entwicklungsländern (Abb. 2) zeigt, daß regenerative Energien an Bedeutung gewinnen werden und müssen, denn der steigende Weltenergieverbrauch als Folge des starken Bevölkerungswachstums und des Strebens nach einem verbesserten Lebensstandard in den Entwicklungsländern kann durch fossile Energieträger allein nicht befriedigt werden.

Aus Abb. 3 ist zu entnehmen, daß die $\mathrm{CO}_{2}$-Emissionen dem Energieszenario „Nachhaltiges Wachstum“ folgen und zwischen 2020 und 2040 ihren Höhepunkt erreichen. Schon dieses Szenario kann zu Temperaturveränderungen von $1,5^{\circ} \mathrm{C}$ führen. Bei kontinuierlicher Fortschreibung heutigen Tuns landet man in einer krisenhaften Zuspitzung in der zweiten Hälfte des nächsten Jahrhunderts.

Viele Skeptiker halten die Chancen, $50 \%$ des Energieverbrauchs der Welt durch regenerative Energien in $2050 \mathrm{zu}$ befriedigen, für visionär. Ein Blick zurück zeigt uns die langen Lernkurven der Energieträger. Noch vor 75 Jahren gab es Benzin nur in der Apotheke
(Abb. 4). Die Zukunft aber gehört den erneuerbaren Energien, davon ist Shell überzeugt. Sicher wird die Menschheit noch für lange Zeit auf Erdöl, Erdgas, Steinkohle, Kernenergie und Braunkohle angewiesen sein. Bereits heute sind Wind und Biomasse aber schon an der Grenze der Wirtschaftlichkeit. Eine der Säulen der regenerativen Energien kann die Photovoltaik werden.

Die Photovoltaik ist allerdings mit DM 1,60 bis DM 1,80 noch weit entfernt von jeder Wirtschaftlichkeit bei einer Netzeinspeisung in Europa. Ganz anders ist bereits heute die Situation in vielen dünn besiedelten, sich entwickelnden Staaten. Heute sind immer noch 2 Mrd. Menschen nicht an ein Stromnetz angeschlossen und dies wird auf absehbare Zeit zu vernünftigen Preisen nicht möglich sein. Inselsysteme werden diesen Menschen eine Mindestversorgung an Strom auf regenerativer Basis ermöglichen (Abb. 5). Die Stromentstehungskosten sind bei weitem günstiger als die Kosten eines Netzanschlusses. So kommt es nicht von ungefähr, daß der weltweite Modulabsatz, insbesondere in den letzten zwei Jahren, drastisch gestiegen ist (Abb. 6). Für 1997 liegt der geschätzte Mittelwert bei etwa $120 \mathrm{MW}$.

Basierend auf den Shell-Szenarien wird im Jahr 2010 die jährliche Nachfrage nach Photovoltaikkomponenten bei 1,5 GW bis $2 \mathrm{GW}$ Kapazität weltweit liegen. Bis zu diesem Zeitpunkt wird mit einem durchschnittlichen Marktwachstum von $22 \%$ pro Jahr gerechnet (Abb. 7). Das erscheint mutig, doch die Europäische Kommission ist in ihrem Weißbuch von 1997 noch mutiger gewesen. Sie peilt eine installierte Leistung für das Jahr 2010 von $3 \mathrm{GW}$ oder $3000 \mathrm{MW}$ allein in Europa an, was ein jährliches Wachstum von $30 \%$ voraussetzt. Eine derartige Expansion ist aber nur möglich, wenn eine kontinuierliche Kostenreduktion erreicht wird.

Was muß getan werden, um dahin zu kommen? Die heutige Technik wird nicht die des Jahres 2010 sein. Deshalb beteiligen sich viele Unternehmen aktiv an der Optimierung der Siliziumzelle, z. B. der Dünnschichtzelle oder der Entwicklung neuer Zellenkonzepte wie der Farbstoffzelle auf Rutheniumbasis, der sogenannten Nanosolarzelle. Vor allen Dingen aber müssen die Produktionskosten reduziert werden, um Photovoltaik wettbewerbsfähig zu machen. Shell geht von einer Kostenreduzierung von $6 \%$ pro Jahr aus.

Dabei ist die gesamte Wertschöpfungskette von der Rohsiliziumherstellung bis zur Modulproduktion zu erfassen. Die Kostenblöcke ergeben sich aus Abb. 8.

Der Produktionsschritt Zellenherstellung wird schon in den nächsten Jahren durch Kostensenkungen geprägt sein. Viele Wettbewerber errichten zur Zeit hochautomatisierte Zellenfabrikationen mit $15 \mathrm{MW}$ bis $25 \mathrm{MW}$ Jahresproduktionskapazität. Allein in Deutschland 


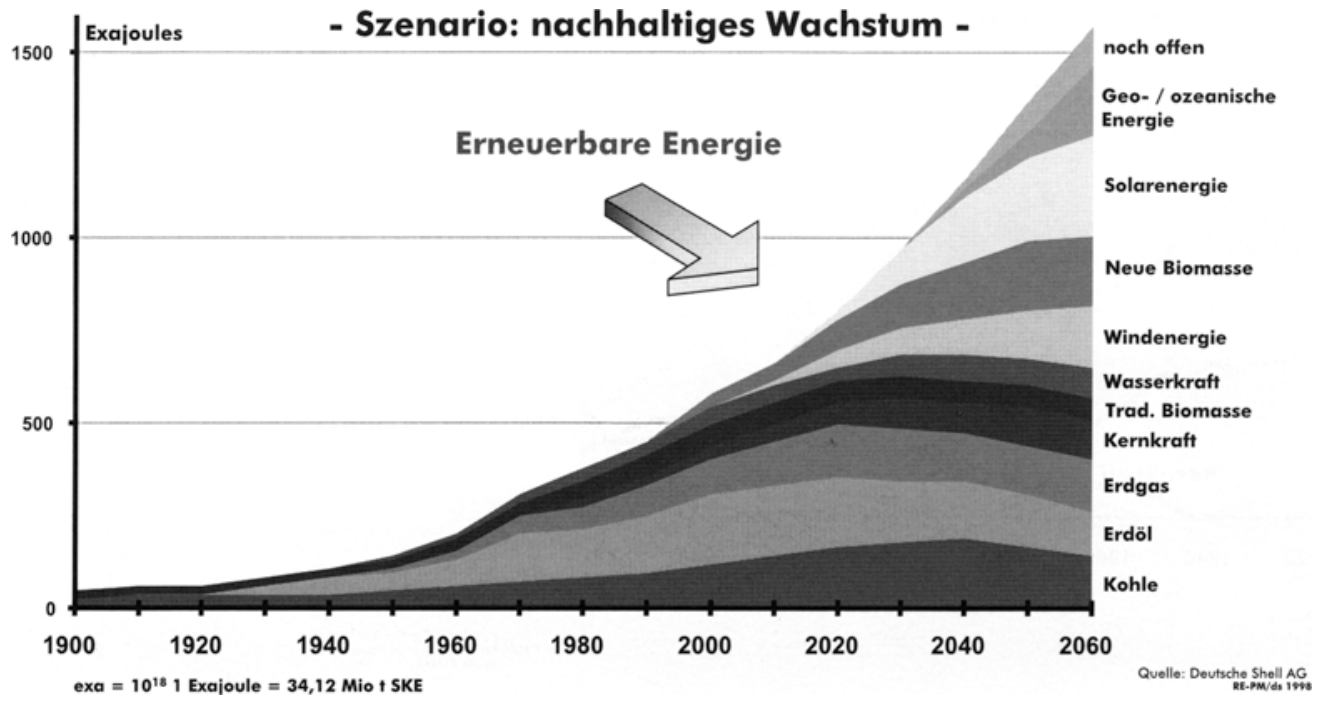

Abb. 1. Szenario: Nachhaltiges Wachstum (Weltenergieverbrauch bis 2060)

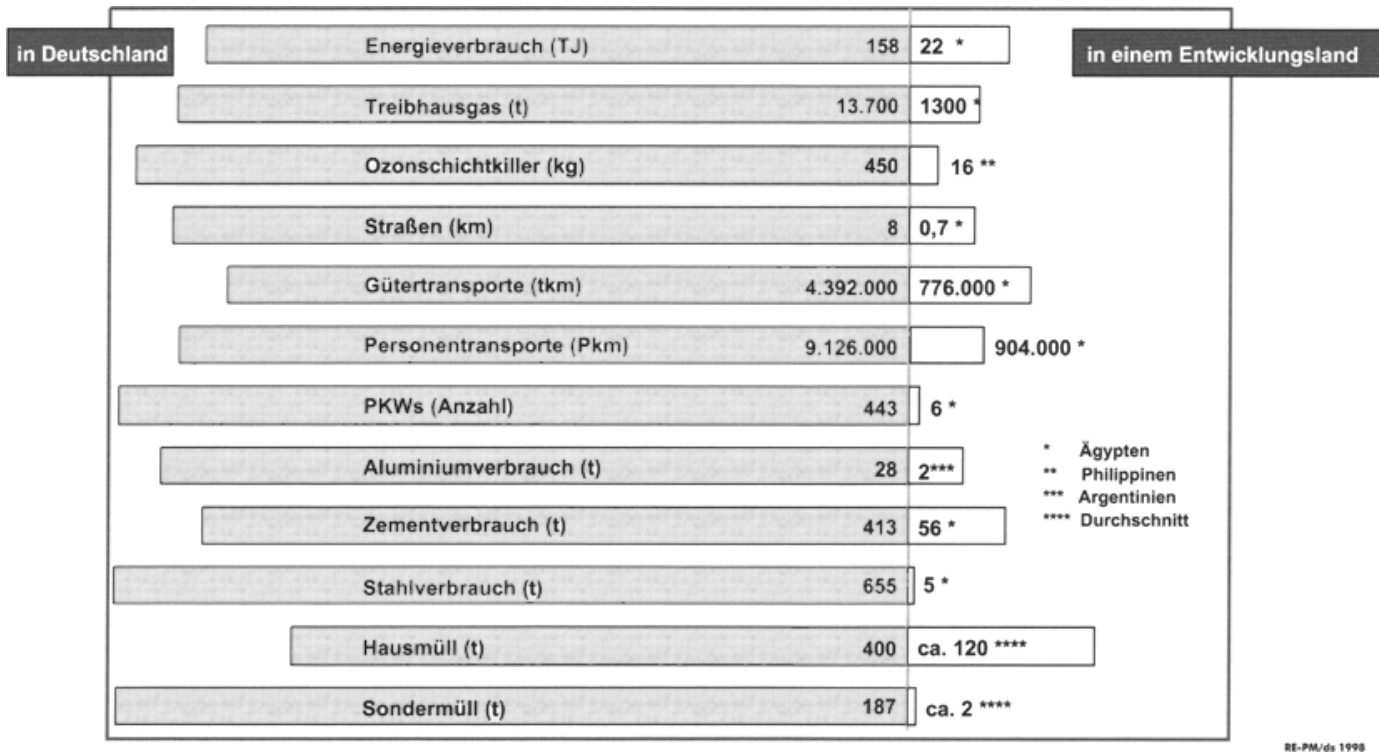

Abb. 2. Jahres-Umweltbelastung durch 1000 Menschen

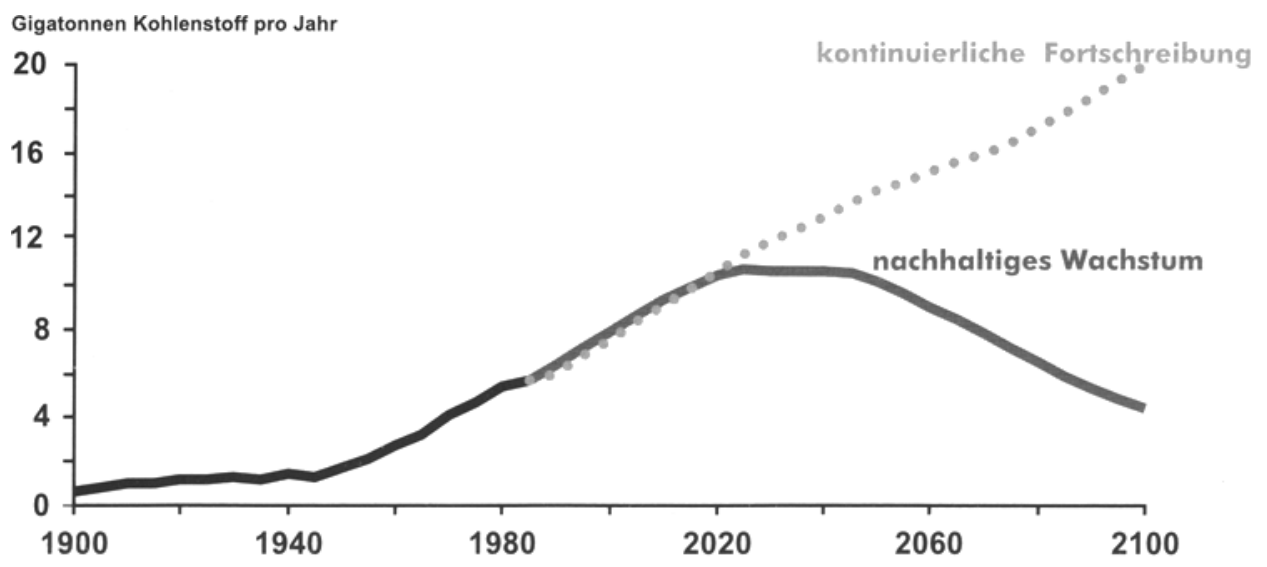

Abb. 3. $\mathrm{CO}_{2}$-Emissionen fossiler Energieträger (1900-2100) 


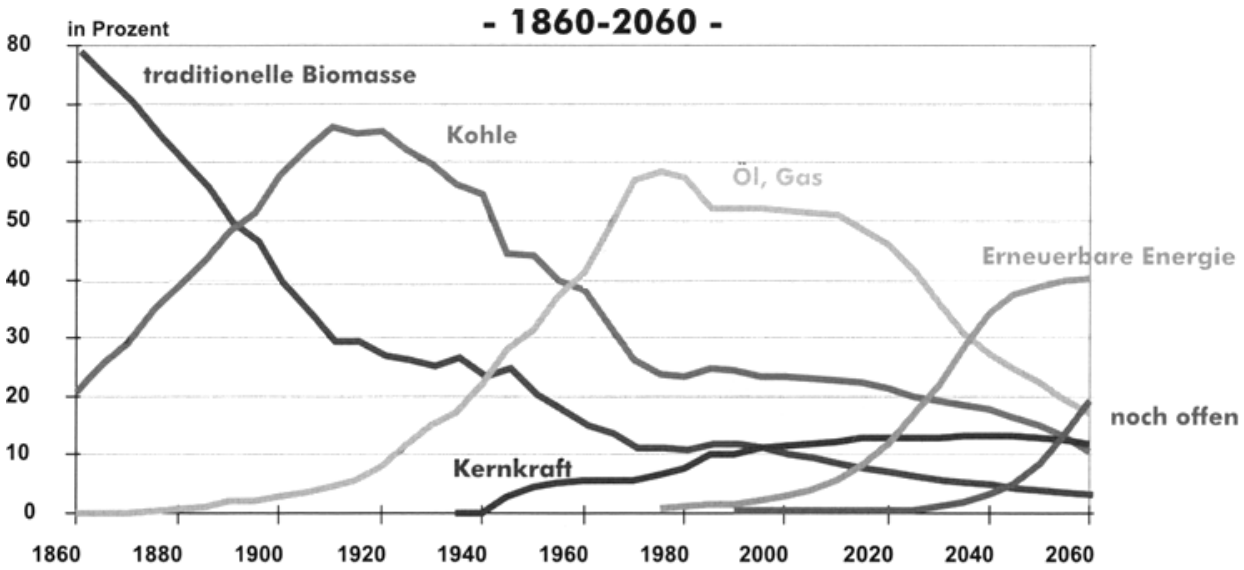

Abb. 4. Lebenszyklen von Energiequellen (1860-2060)

\section{Solar Control System}

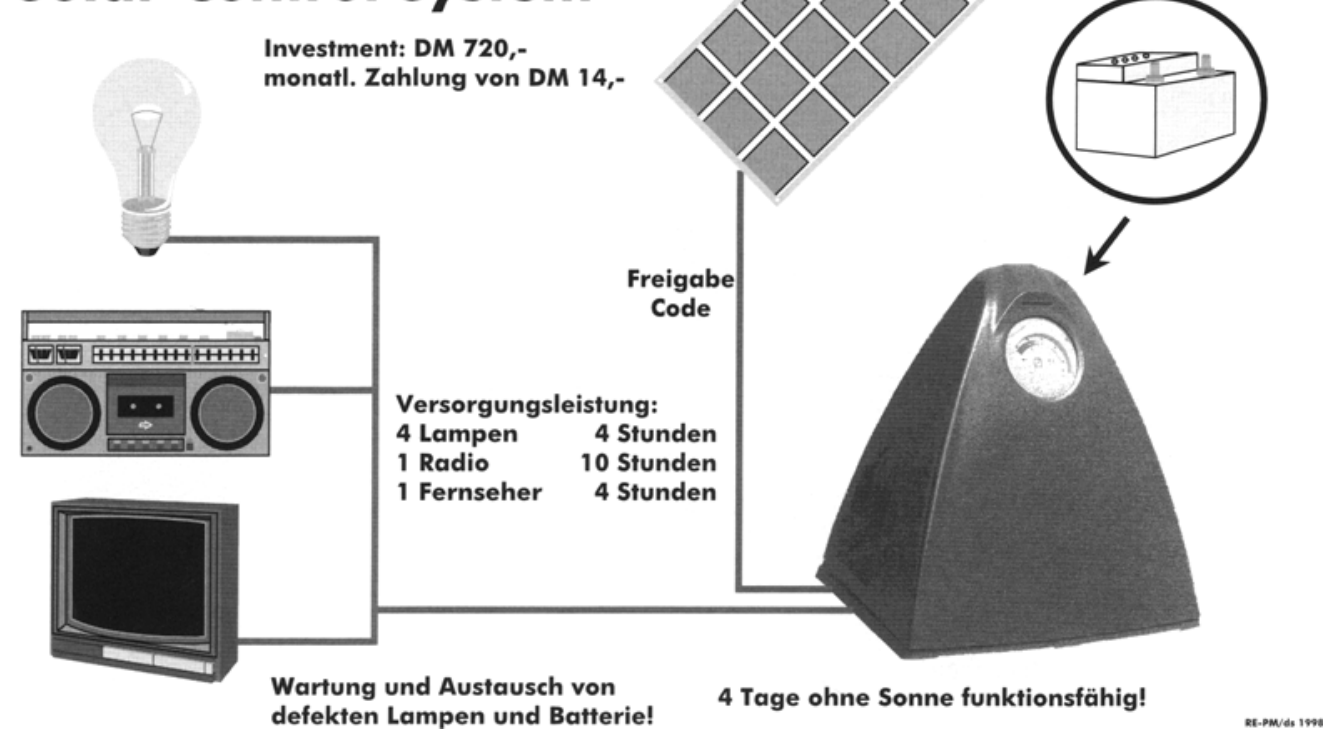

Abb. 5. Solar Control-System

sind drei Investitionsprojekte im Bau. Nach der Optimierung der Wafer- und Zellenproduktion ist auch die Kostensenkung bei der Modul- und Systemproduktion in Angriff zu nehmen. Ein 100000-Dächer-Programm in Deutschland würde hier für diesen Schritt ebenfalls eine Signalfunktion haben.

Heute liegt der Anteil der Photovoltaik an der Stromerzeugung in Deutschland bei ca. $0,004 \%$. Selbst bei einem angenommenen jährlichen Wachstum von $30 \%$ würde der Anteil des photovoltaisch erzeugten Stroms im Jahre 2010 erst bei ca. 0,13 \% liegen. Damit ist klar: Photovoltaik kann unsere Umwelt- und Energieprobleme kurzfristig nicht lösen.
Ohne gleichzeitige Energieeinsparung wird Deutschland die angestrebte Minderung der $\mathrm{CO}_{2}$-Emissionen nicht erreichen. Braunkohle, Steinkohle und Kernenergie werden jedoch auch in den nächsten 30 Jahren die Stromversorgung, insbesondere im Grundlastbereich, sicherstellen müssen. Die günstigen Gestehungskosten für die Elektrizitätsgewinnung aus diesen klassischen Energieträgern bleiben eine Voraussetzung für das Abfedern der zusätzlichen Kosten, die mit der Bereitstellung regenerativer Energien verbunden sind.

Um den Unternehmen, die auf dem Gebiet der erneuerbaren Energien tätig sind, eine vernünftige Planungsgrundlage an die Hand zu geben, muß in Deutschland - 


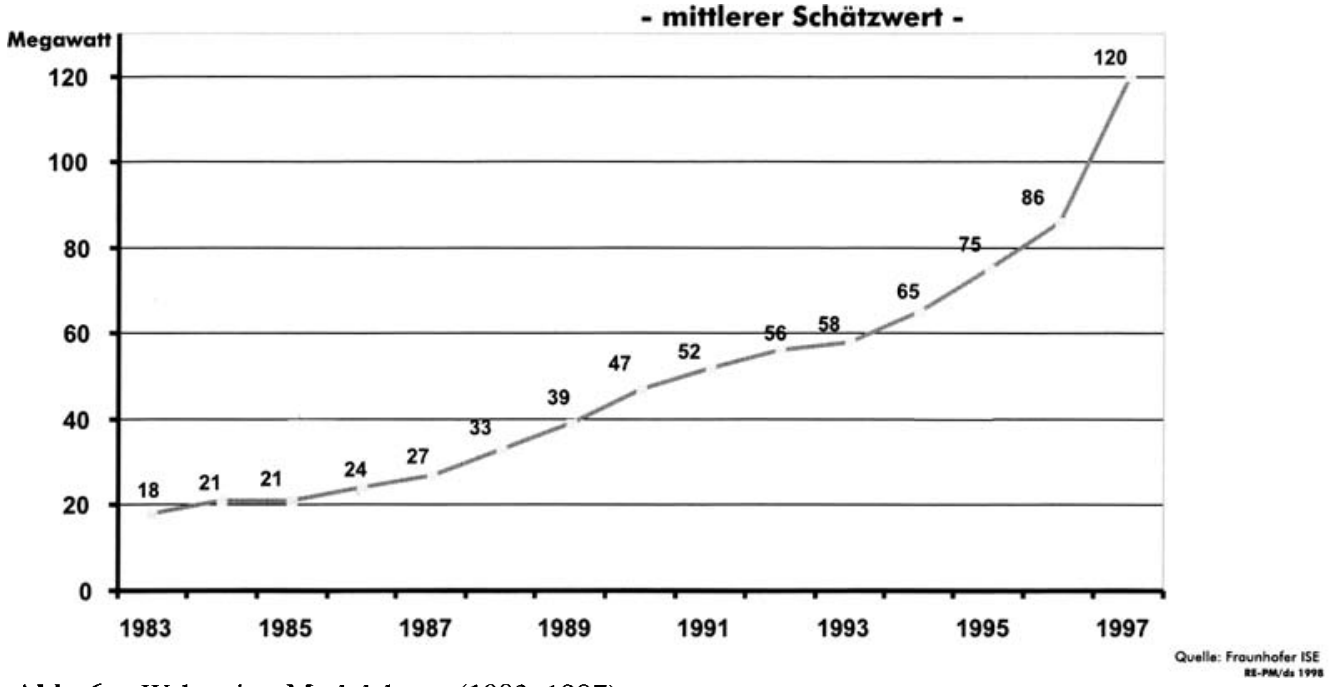

Abb. 6. Weltweiter Modulabsatz (1983-1997)

\section{globales Marktvolumen und Preisentwicklung bis zum Jahr 2010}

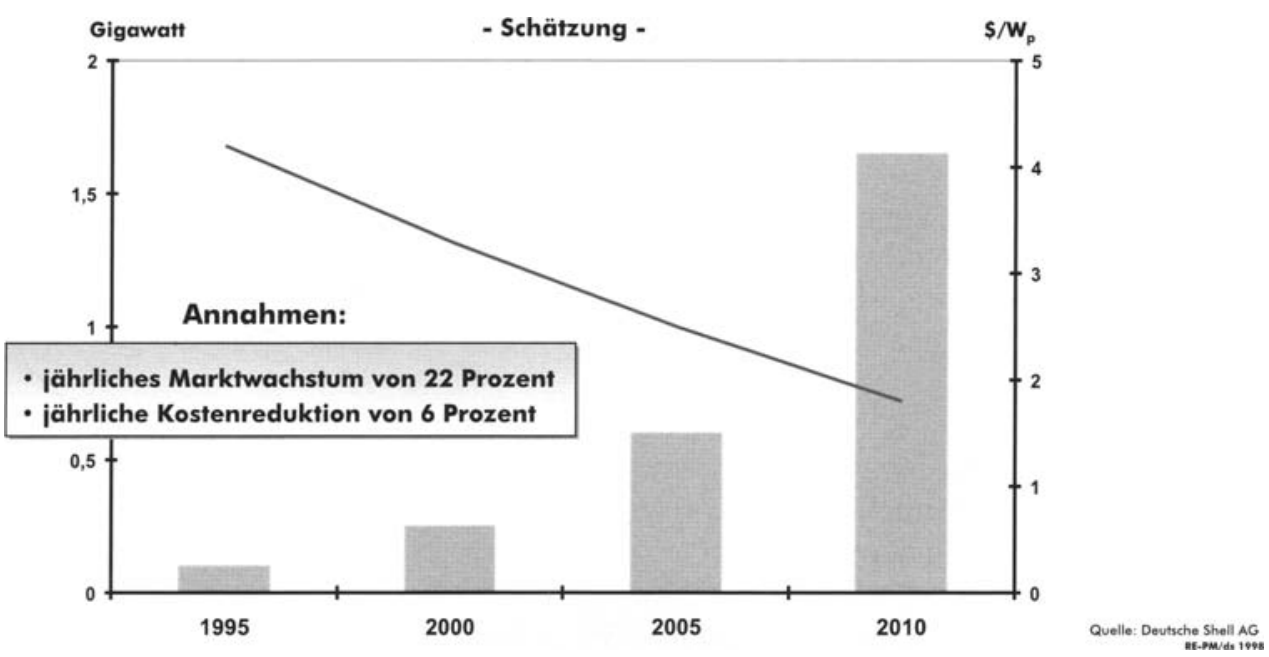

Abb. 7. Globales Marktvolumen und Preisentwicklung von PV-Panels bis 2010

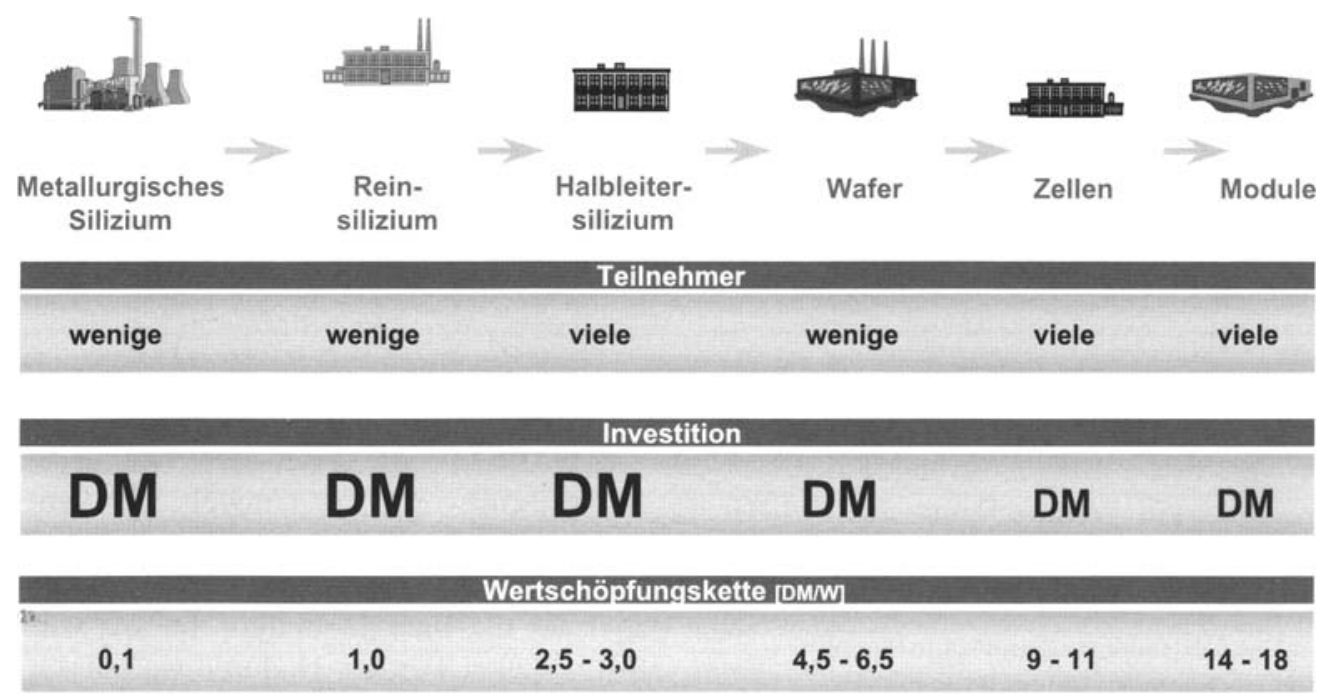

Abb. 8. Silizium-Produktionskette 
besser noch in Europa - endlich eine klare, konsistente Politik betrieben werden. Im Vordergrund müssen stehen:

(1) Förderung von Forschung und Entwicklung an den Universitäten sowie in den Unternehmen.

(2) Erarbeitung einer Markteinführungsstrategie, die auch die Rolle der öffentlichen Hand als wichtigen Nachfrager klärt.
(3) Einheitliche und auskömmliche Einspeisevergütungen für Strom aus regenerativen Energiequellen. Als Alternative ist die Vorgabe eines stetig wachsenden, verbindlichen Anteils von „ÖkoStrom" an der Stromversorgung denkbar.

Dann ist eine Realisierung der Ziele der EU-Kommission im Bereich der Photovoltaik sicher erreichbar.

\title{
Jugendwettbewerb für Sonnenkinder
}

\author{
G. Becker ${ }^{1}$
}

\begin{abstract}
Unser Ziel, die Jugend für eine Partnerschaft für die Zukunft zu gewinnen, haben wir im ersten Versuch sehr gut erreicht. Die Arbeiten der Jugend zeichnen sich durch Kreativität, Professionalität und ein besonders starkes Einfühlungsvermögen im Denken mit der Sonne aus. Der Youth Award wurde an fünf Gruppen in den Bereichen gemeinsame Initiative von Lehrern und Schülern, Gesamtenergiekonzept für Ladakh, elektrische Energie für Nepal, architektonische Entwürfe für eine Schule und Solarcontainer - verliehen.
\end{abstract}

Schlüsselwörter: 2. Weltkonferenz für Photovoltaik; Photovoltaikdemonstrationsprojekte der Jugend; Hybridlösungen mit Photovoltaik, Wind, Wasser und Sonnenkollektoren; Container-Kleinwasserkraftwerk; Modulare Energieversorgungssysteme; Photovoltaikcontainer

Youth Award for solar youth groups. We achieved our target to win the youth for a partnership for the future very well in the first attempt. The works of youth distinguish themselves through creativity, professionalism and a strong intuition in thinking with the sun. The Youth Award was given to five groups in the fields: common initiative of teachers and pupils, total energy concept for Ladakh, electric energy for Nepal, architectural drafts for a school and solar containers.

Keywords: 2nd world conference on photovoltaics; photovoltaic demonstration projects of the youth; hybrid solutions with photovoltaics, wind, water and thermal collectors; container-small hydro electric power plant; modular energy supply system; photovoltaic container

Prof. Schmid, der Präsident der 2. Weltkonferenz für Photovoltaik, Prof. Friedrich von der Universität Graz und Ing. Gernot Becker vom österr. Bundesverband für Photovoltaik hatten die Idee, zur Weltkonferenz auch jene Gruppe einzuladen, die aus heutiger Sicht die

\footnotetext{
' Ing. Gernot Becker, Vorstand des Bundesverbandes für Photovoltaik der Bundeswirtschaftskammer Österreichs, ATB-Antennen, Umwelt, Technik, Becker, Dörferstraße 16, A-6067 Absam.
}

größte Last der Umweltverschmutzungen zu tragen haben wird - die Jugend.

Wir als aktive Befürworter der Umwelttechniken insgesamt und der Photovoltaik im speziellen versuchen eine Trendwende hin zu einer lebenswerteren Welt und setzen dabei auf die Energiequellen, die uns von der Natur selbst gegeben werden. Dadurch wollen wir der Natur helfen, ihre eigene Kraft der Selbstreinigung zu verstärken. 